\title{
Calculation of size-Intensive transition moments from the coupled cluster singles and doubles linear response function
}

\author{
Henrik Koch, a) Rika Kobayashi, Alfredo Sanchez de Merás, b) and Poul Jørgensen \\ Department of Chemistry, Aarhus University, DK-8000 Aarhus C. Denmark
}

(Received 20 October 1993; accepted 16 November 1993)

\begin{abstract}
Coupled cluster singles and doubles linear response (CCLR) calculations have been carried out for excitation energies and dipole transition strengths for the lowest excitations in $\mathrm{LiH}, \mathrm{CH}^{+}$, and $\mathrm{C}_{4}$ and the results compared with the results from a $\mathrm{CI}$-like approach to equation of motion coupled cluster (EOMCC). The transition strengths are similar in the two approaches for single molecule calculations on small systems. However, the CCLR approach givcs sizc-intensive dipole transition strengths, while the EOMCC formalism does not. Thus, EOMCC calculations can give unphysically dipole transition strengths, e.g., in EOMCC calculations on a sequence of noninteracting $\mathrm{LiH}$ systems we obtained a negative dipóle strength for the lowest totally symmetric dipole allowed transition for 19 or more noninteracting LiH systems. The CCLR approach is shown to be a very attractive "black box" approach for the calculation of transition moments.
\end{abstract}

\section{INTRODUCTION}

The calculation of molecular properties and excitations can be performed efficiently using response function approaches. Linear response (LR) functions determine frequency dependent second-order molecular properties for the reference state, and the poles and residues of linear response functions determine transition energies and firstorder transition matrix elements, respectively, from the reference state to excited states. ${ }^{1,2}$ Linear response functions have been derived for self-consistent field (SCF), ${ }^{3}$ multiconfiguration self-consistent field (MCSCF), ${ }^{4}$ and coupled cluster (CC) wave functions. ${ }^{5,6}$ Linear response functions have also been derived for perturbation based approaches such as the second-order polarization propagator approach (SOPPA $)^{7}$ and for approaches based on second-order Møller-Plesset perturbation (MP2) theory. ${ }^{8,9}$ Except for the $\mathrm{CC}$ response functions the above response functions have been implemented and have routinely been used to describe molecular properties.

The coupled cluster singles and doubles (CCSD) model is one of the most promising approaches for accurate $a b$ initio electronic structure calculations. ${ }^{10}$ It describes equilibrium structures of closed shell molecules more accurately than the wave functions for which response functions have been implemented. Finite difference calculations of static second-order molecular properties also indicate that the CCSD model describes these properties very well. ${ }^{11}$ It is therefore expected that the CCSD linear response function will give accurate frequency dependent second-order molecular properties and first-order transition matrix elements. In this paper we describe the first implementation of the CCSD linear response function for the calculation of first-order transition moments.

The CC linear response function was first derived by

\footnotetext{
a) Present address: UNI.C, Olof Palmes Allé 38, 8200 Århus N, Denmark. b) Present address: Universidad de Valencia, Departamento de Quimica Fisica, Doctor Moliner, 50 Burjasot (Valencia), Spain.
}

Monkhorst, ${ }^{5}$ and Monkhorst and Dalgaard. ${ }^{6}$ A simpler and more general derivation which included a derivation of the quadratic response function has been presented by Koch and Jørgensen. ${ }^{12}$ Calculations of excitation energies from the CCSD linear response function were first carried out by Koch et al. ${ }^{13}$

Finite field CCSD calculations of static second-order molecular properties are usually carried out using field relaxed SCF orbitals. The CC linear response functions are derived without this field relaxation. The relaxation of the orbitals must be neglected in frequency dependent calculations because the field dependence of the orbitals introduces poles at the excitation energies of the noncorrelated system. These poles are nonphysical for a correlated calculation and they make the calculation ill-defined for frequencies in regions close to these poles.

Molecular properties which scale with the size of the system are called size-extensive properties, whereas molecular properties which are independent of the size of the system are called size-intensive properties. ${ }^{14}$ In Ref. 13 it was shown that the CCLR excitation energies are sizeintensive. We show in this paper that CCLR transition moments are size-intensive and that CCLR second-order molecular properties are size-extensive.

Stanton and Bartlett ${ }^{15}$ have recently derived a CI-like approach to the equation of motion coupled cluster (EOMCC) formalism for the evaluation of excitation energies and transition matrix elements. The excitation energies in the EOMCC and CCLR approach are identical, but they offer different approximations in approximate calculations of molecular properties, for example transition moments. In the full configuration interaction (FCI) limit where no truncation is carried out of the projection manifold, the two approaches both give the FCI transition strength (the square of the transition moment). In approximate calculations such as the reported $\mathrm{CC}$ singles and doubles calculations, the results of the two approaches differ; the EOMCC transition moments are not size-intensive. This can lead to absurd transition strength values in the 
EOMCC formalism. For example, in an EOMCC calculation on noninteracting $\mathrm{LiH}$ systems, we obtained a negative transition strength with 19 or more noninteracting $\mathrm{LiH}$ systems. For a single LiH system the EOMCC and the CCLR results are very similar.

In Sec. II we summarize the derivation of the CC linear response function and describe the implementation of the CCSD linear response function for the calculation of first-order transition matrix elements. We also demonstrate in Sec. II that the transition moments in the CCLR approach are size-intensive. In Sec. III the EOMCC method is expressed in terms of the notation we have used for CCLR. We show that the EOMCC transition moments are not size-intensive. Section IV contains numerical applications to $\mathrm{LiH}, \mathrm{CH}^{+}$, and $\mathrm{C}_{4}$, and in Sec. $\mathrm{V}$ we give some concluding remarks.

\section{THEORY}

\section{A. Transition moments from the linear response function}

Following the notation of Koch and J $\phi$ rgensen, ${ }^{12}$ the $\mathrm{CC}$ linear response function at frequency $\omega$ becomes [see Eq. (94) of Ref. 12]

$$
\begin{aligned}
\langle\langle C ; D\rangle\rangle_{\omega}= & \sum_{\mu}\left\langle\Lambda\left|\left[C, \tau_{\mu}\right]\right| \mathrm{CC}\right\rangle X_{\mu}^{D}(\omega) \\
& +\sum_{\mu}\left\{\left\langle\Lambda\left|\left[D, \tau_{\mu}\right]\right| \mathrm{CC}\right\rangle\right. \\
& \left.+\sum_{\gamma}\left\langle\Lambda\left|\left[H, \tau_{\mu}\right], \tau_{\gamma}\right| \mathrm{CC}\right\rangle X_{\gamma}^{D}(\omega)\right\} X_{\mu}^{C}(-\omega),
\end{aligned}
$$

where $C$ and $D$ are operators, $H$ the zero-order BornOppenheimer Hamiltonian, and $|\mathrm{CC}\rangle$ is the coupled cluster wave function

$$
|\mathrm{CC}\rangle=\exp (T)|\mathrm{HF}\rangle .
$$

The cluster operator in Eq. (2) for an $N$ electron system is

$$
T=T_{1}+T_{2}+\cdots+T_{N},
$$

where

$$
\begin{aligned}
& T_{1}=\sum_{a i} t_{i}^{a} E_{a i}, \\
& T_{2}=\sum_{(a i)}>(b j) \\
& t_{i j}^{a b} E_{a i} E_{b j}
\end{aligned}
$$

are the one- and two-electron cluster operators and $E_{a i}$ are generators of the unitary group. The indices $i j k l$ and $a b c d$ refer to occupied and unoccupied orbitals in the HartreeFock reference state $|H F\rangle$. Using a shorthand notation, the cluster operator becomes

$$
T=\sum_{\mu} t_{\mu} \tau_{\mu},
$$

where $t_{\mu}$ denotes the cluster amplitudes and $\tau_{\mu}$ the corresponding excitation operators. The $\mathrm{CC}$ amplitudes are determined from the $\mathrm{CC}$ amplitude equations

$$
\langle\mu|\exp (-T) H| C C\rangle=0,
$$

where

$$
\langle\mu|=\langle\mathrm{HF}| \tau_{\mu}^{+} .
$$

The state $\langle\Lambda|$ is defined as

$$
\langle\Lambda|=\langle\mathrm{HF}|+\sum_{\mu} \xi_{\mu}\langle\mu| \exp (-T)
$$

and the $\zeta_{\mu}$ parameters in Eq. (9) are determined from the set of linear equations

$$
\xi^{T} \mathbf{A}=\eta^{T}
$$

with $\mathbf{A}$ as the CC Jacobian

$$
A_{\mu \nu}=\left\langle\mu|\exp (-T)|\left[H, \tau_{v}\right] \mid \mathrm{CC}\right\rangle
$$

and the elements of the right-hand side row vector are defined as

$$
\eta_{v}=-\left\langle\mathrm{HF}\left|\left[H, \tau_{v}\right]\right| \mathrm{CC}\right\rangle .
$$

The vectors $X_{\mu}^{D}(\omega)$ and $X_{\mu}^{C}(-\omega)$ are solutions to sets of linear equations, which for $X^{D}(\omega)$ are

$$
\begin{aligned}
& (-\mathbf{A}+\omega \mathbf{1}) X_{v}^{D}(\omega)=\xi_{v}^{D}, \\
& \xi_{v}^{D}=\langle v|\exp (-T) D| C C\rangle .
\end{aligned}
$$

We have assumed that the calculations are carried out using an orthogonal basis where the metric is the unit matrix

$$
S_{\mu \nu}=\left\langle\mu\left|\exp (-T) \tau_{v}\right| \mathrm{CC}\right\rangle=\left\langle\mu\left|\tau_{v}\right| \mathrm{HF}\right\rangle .
$$

When $C$ and $D$ are components of the dipole operator, Eq. (1) determines the components of the frequency dependent polarizability. In Ref. 16 we have described an implementation of the CCSD frequency dependent polarizability.

Excitation energies appear at the poles of the $\mathrm{CC}$ linear response function and are determined as the eigenvalues $\omega_{k}$ of the nonsymmetric coupled cluster Jacobian. Since the Jacobian is nonsymmetric, we have both right $(|k\rangle)$ and left $(\langle k|)$ eigenstates

$$
\begin{aligned}
& \left(\mathbf{A}-\omega_{k} \mathbf{1}\right) X_{k}^{R}=0, \\
& \left(\mathbf{A}^{T}-\omega_{k} \mathbf{1}\right) X_{k}^{L}=0,
\end{aligned}
$$

and we assume that these eigenvectors are normalized:

$$
\langle k \mid k\rangle=\left(X_{k}^{L}\right)^{T} X_{k}^{R}=1 .
$$

The transition matrix element from the reference state $|0\rangle$ to the excited state $|k\rangle$ is determined from the residue of the CC linear response function [see Eq. (102) of Ref. 12]

$$
\begin{aligned}
\langle k|D| 0\rangle= & \sum_{\mu} X_{\mu k}^{L}\langle\mu|\exp (-T) D| C C\rangle \\
\langle 0|C| k\rangle= & \sum_{\mu}\left\langle\Lambda\left|\left[C, \tau_{\mu}\right]\right| C C\right\rangle X_{\mu k}^{R}+\sum_{\nu \mu} X_{\nu}^{C}\left(-\omega_{k}\right) \\
& \times\left\langle\Lambda\left|\left[\left[H, \tau_{v}\right], \tau_{\mu}\right]\right| C C\right\rangle X_{\mu k}^{R}
\end{aligned}
$$


The transition matrix elements $\langle 0|C| k\rangle$ and $\langle k|C| 0\rangle$ are not the adjoint of each other as the Schrödinger equation in $\mathrm{CC}$ theory is solved using a projection technique. The evaluation of the transition matrix elements require that Eqs. (16) and (17) are solved for the eigenvalue $\omega_{k}$ and eigenvectors $X_{k}^{L}$ and $X_{k}^{R}$, and that one set of linear equations of the structure in Eq. (13) is solved to obtain $X_{v}^{C}\left(-\omega_{k}\right)$. Furthermore, the $\zeta$ parameters have to be determined from Eq. (10).

The evaluation of the $\mathrm{CC}$ linear response function $\langle\langle C ; D\rangle\rangle_{\omega}$ and the transition matrix elements $\langle 0|C| k\rangle$ and $\langle k|D| 0\rangle$ have much in common. In both cases, the linear equations in Eq. (10) and in Eq. (13) must be solved. This rcquires the linear transformation

$$
\begin{aligned}
& u_{v}=\sum_{\mu} b_{\mu} A_{\mu v}, \\
& m_{\mu}=\sum_{\mu} A_{\mu v} b_{v}
\end{aligned}
$$

for which iterative techniques are used. In these equations $\mathbf{b}$ is a trial vector and $\mathbf{u}$ and $\mathbf{m}$ are linear transformed vectors with the Jacobian as transformation matrix. Equations (21) and (22) are not identical as the Jacobian $\mathbf{A}$ is nonsymmetric. The evaluation of the linear transformations in Eqs. (21) and (22) enters the evaluation of the CCSD molecular Hessian. In Ref. 17 we have described an implementation of the CCSD molecular Hessian and in that paper details are given about our implementation of Eqs. (21) and (22).

The evaluation of the linear response function and the transition matrix elements further have in common terms of the structure

$$
\begin{aligned}
& \left\langle\Lambda\left|\left[C, \sum_{\mu} b_{\mu} \tau_{\mu}\right]\right| C C\right\rangle, \\
& \sum_{\mu} b_{\mu}\langle\mu|\exp (-T) D| C C\rangle, \\
& \sum_{\mu \nu}\left\langle\Lambda\left|\left[\left[H_{0}, \tau_{\mu}\right], \tau_{\nu}\right]\right| \mathrm{CC}\right\rangle b_{\mu} c_{\nu} .
\end{aligned}
$$

Such terms also appear in the evaluation of the CCSD molecular Hessian, and we refer to Ref. 17 where an implementation of these terms is described. The evaluation of Eqs. (23) and (24) was carried out in Ref. 17 with $C$ and $D$ referring to two-electron operators. When $C$ and $D$ are one-electron operators as in this case their evaluation simplifies significantly. As stated previously, the metric is assumed to be a unit matrix. This is obtained using an orthogonal basis

$$
\begin{aligned}
& \tau_{1}=\left\{\frac{1}{\sqrt{2}} E_{a i}\right\}, \\
& \tau_{2 s}=\left\{\frac{1}{2 \sqrt{\left(1+\delta_{i j}\right)\left(1+\delta_{a b}\right)}}\left(E_{a i} E_{b j}+E_{a j} E_{b i}\right) \mid a \geqslant b, \geqslant j\right\}, \\
& \tau_{2 t}=\left\{\frac{\sqrt{3}}{6}\left(E_{a i} E_{b j}-E_{a j} E_{b i}\right) \mid a>b, i>j\right\},
\end{aligned}
$$

where $\tau_{2 s}$ and $\tau_{2 t}$ denote the singlet-singlet and triplettriplet spin coupled double excitation manifolds where spin couplings initially are carried out on the occupiedoccupied and unoccupied-unoccupied orbital indices.

The linear transformations in Eqs. (21) and (22) and the terms in Eqs. (23)-(25) are most conveniently implemented in the elementary basis

$$
\begin{aligned}
\tau_{1} & =\left\{E_{a i}\right\}, \\
\tau_{2} & =\left\{E_{a i} E_{b j} \mid a i \geqslant b j\right\}
\end{aligned}
$$

and in our calculation of the CCSD transition moments we transform vectors between the orthogonal and the elementary basis and vice versa whenever convenient.

\section{B. Size intensivity of the transition moments}

A size-extensive molecular property scales with the size of the system and a size-intensive molecular property is independent of the size of the system. We have previously shown that excitation energies in the CCLR model are size-intensive. We will now show that the transition moments are size-intensive and the second-order molecular properties are size-extensive. To do so, we need to prove that a CCLR calculation on a system consisting of subsystems $A$ and $B$ at infinite separation give the transition moments of the subsystems, and that second-order molecular properties are the sums of the second-order molecular properties of the subsystems.

Since the subsystems are at infinite separation the Hamiltonian of the combined system reduces to the sum of the Hamiltonians for the subsystems

$$
H=H_{\mathrm{A}}+H_{\mathrm{B}} \text {. }
$$

The coupled cluster reference wave function is sizeextensive and the cluster operator therefore can be written as the sum of the cluster operators of the two subsystems

$$
T=T_{\mathrm{A}}+T_{\mathrm{B}} \text {. }
$$

The excitation operators $\left\{\tau_{v}\right\}$ can be divided into a set referencing only subsystem $\mathrm{A}\left\{\tau_{\mathrm{A}}\right\}$, a set referencing only subsystem $B\left\{\tau_{B}\right\}$, and a set referencing both $A$ and $B$ $\left\{\tau_{A B}\right\}$. The projection manifold may analogously be expressed in terms of excitations for subsystem $A$, $\left(\left\langle\mathrm{HF}_{\mathrm{B}} \mathrm{HF}_{\mathrm{A}}\right| \tau_{\mathrm{A}}^{+}\right)$, for subsystem $\mathrm{B}\left(\left\langle\mathrm{HF}_{\mathrm{B}} \mathrm{HF}_{\mathrm{A}}\right| \tau_{\mathrm{B}}^{+}\right)$, and excitations that reference the two subsystems $\left(\left\langle\mathrm{HF}_{\mathrm{B}} \mathrm{HF}_{\mathrm{A}}\right| \tau_{\mathrm{AB}}\right)$. The Jacobian can be written in a threecomponent form with the projection manifold $\left\{\left\langle\mathrm{HF}_{\mathrm{B}} \mathrm{HF}_{\mathrm{A}}\right| \tau_{\mathrm{A}}^{+},\left\langle\mathrm{HF}_{\mathrm{B}} \mathrm{HF}_{\mathrm{A}}\right| \tau_{\mathrm{B}}^{+},\left\langle\mathrm{HF}_{\mathrm{B}} \mathrm{HF}_{\mathrm{A}}\right| \tau_{\mathrm{AB}}^{+}\right\}$constituting the row labels and the excitation operators $\left\{\tau_{\mathrm{A}}, \tau_{\mathrm{B}}\right.$, $\left.\tau_{\mathrm{AB}}\right\}$ constituting the column labels

$$
\begin{aligned}
\mathbf{A} & =\left(\begin{array}{ccc}
\mathbf{A}^{\mathrm{A}, \mathrm{A}} & \mathbf{A}^{\mathrm{A}, \mathrm{B}} & \mathbf{A}^{\mathrm{A}, \mathrm{AB}} \\
\mathbf{A}^{\mathrm{B}, \mathrm{A}} & \mathbf{A}^{\mathrm{B}, \mathrm{B}} & \mathbf{A}^{\mathrm{B}, \mathrm{AB}} \\
\mathbf{A}^{\mathrm{AB}, \mathrm{A},} & \mathbf{A}^{\mathrm{AB}, \mathrm{B},} & \mathbf{A}^{\mathrm{AB}, \mathrm{AB}},
\end{array}\right) \\
& =\left(\begin{array}{ccc}
\mathbf{A}^{\mathrm{A}, \mathrm{A}} & 0 & \mathbf{A}^{\mathrm{A}, \mathrm{AB}} \\
0 & \mathbf{A}^{\mathrm{B}, \mathrm{B}} & \mathbf{A}^{\mathrm{B}, \mathrm{AB}} \\
0 & 0 & \mathbf{A}^{\mathrm{AB}, \mathrm{AB}}
\end{array}\right) .
\end{aligned}
$$


$\mathbf{A}^{\mathrm{A}, \mathrm{A}}$ and $\mathbf{A}^{\mathrm{B}, \mathrm{B}}$ are the Jacobian for subsystems $\mathrm{A}$ and $\mathrm{B}$, respectively. The zero blocks follow from the identity

$$
\left[\exp \left(T_{\mathrm{B}}\right), H_{\mathrm{A}}\right]=0
$$

which allows us to integrate the dependence of one of the subsystems. Since the metric is a unit matrix, it follows immediately that the eigenvalues of the CCLR Jacobian satisfy

$$
\left|\mathbf{A}^{\mathrm{A}, \mathrm{A}}-\omega \mathbf{I}^{\mathrm{AA}}\right|\left|\mathbf{A}^{\mathrm{B}, \mathrm{B}}-\omega \mathbf{I}^{\mathrm{BB}}\right|\left|\mathbf{A}^{\mathrm{AB}, \mathrm{AB}}-\omega \mathbf{I}^{\mathrm{AB}, \mathrm{AB}}\right|=0 .
$$

The eigenvalues of the two subsystems are thus eigenvalues of the combined system and the excitation energies in a CCLR calculation are size-intensive as previously shown in Ref. 13.

From the structure of the Jacobian [Eq. (33)] it is seen that the eigenvectors of the subsystems

$$
\mathbf{A}^{\mathrm{A}, \mathrm{A}} X_{k \mathrm{~A}}^{R}=\omega_{k \mathrm{~A}} X_{k \mathrm{~A}}^{R}
$$

also are eigenvectors of the combined system

$$
\mathbf{A} X_{k}^{R}=\omega_{k \mathrm{~A}}^{\mathrm{A}} X_{k}^{R},
$$

where

$$
X_{k}^{R}=\left(\begin{array}{c}
X_{k \mathrm{~A}}^{R} \\
0 \\
0
\end{array}\right)
$$

The structure of the Jacobian also assures that the solution vector in Eq. (13) for the combined system becomes

$$
(-\mathbf{A}+\omega \mathbf{I})\left(\begin{array}{c}
X_{\mathrm{A}}^{C}(\omega) \\
X_{\mathrm{B}}^{C}(\omega) \\
0
\end{array}\right)=\left(\begin{array}{c}
\xi_{\mathrm{A}}^{C} \\
\xi_{\mathrm{B}}^{C} \\
0
\end{array}\right),
$$

where $X_{\mathrm{A}}^{C}(\omega)$ and $X_{\mathrm{B}}^{C}(\omega)$ are the solution vectors for the subsystems. The $\xi$ solution vectors [Eq. (10)] for the combined system becomes

$$
\left(\xi_{\mathrm{A}}, \xi_{\mathrm{B}}, \zeta_{\mathrm{AB}}\right) \mathrm{A}=\left(\eta_{\mathrm{A}}, \eta_{\mathrm{B}}, 0\right),
$$

where

$$
\begin{aligned}
& \xi_{\mathrm{A}} \mathbf{A}^{\mathrm{AA}}=\eta_{\mathrm{A}}, \\
& \xi_{\mathrm{B}} \mathrm{A}^{\mathrm{BB}}=\eta_{\mathrm{B}}, \\
& \xi_{\mathrm{A}} \mathrm{A}^{\mathrm{A}, \mathrm{AB}}+\xi_{\mathrm{B}} \mathbf{A}^{\mathrm{B}, \mathrm{AB}}+\xi_{\mathrm{AB}} \mathbf{A}^{\mathrm{AB}, \mathrm{AB}}=0 .
\end{aligned}
$$

The solution vectors of the subsystems are $\xi_{\mathrm{A}}$ and $\xi_{\mathrm{B}}$, whereas $\xi_{\mathrm{AB}}$ is the solution vector of the linear equation [Eq. (43)]. Thus, the $\zeta$ vector does not separate into components referring only to the subsystems.

Let us first consider the evaluation of the right transition moment in Eq. (19):

$$
\begin{aligned}
\langle k|D| 0\rangle= & \sum_{\mu_{\mathrm{A}}}\left(X_{\mu_{\mathrm{A}} k}^{L}\left\langle\mathrm{HF}_{\mathrm{B}} \mu_{\mathrm{A}}\right| \exp \left(-T_{\mathrm{A}}-T_{\mathrm{B}}\right)\left(D_{\mathrm{A}}+D_{\mathrm{B}}\right)\right. \\
& \left.\times \exp \left(T_{\mathrm{A}}+T_{\mathrm{D}}\right)\left|\mathrm{HF}_{\mathrm{A}} \mathrm{HF}_{\mathrm{D}}\right\rangle\right) \\
= & \sum_{\mu_{\mathrm{A}}} X_{\mu_{\mathrm{A}} k}^{L}\left\langle\mu_{\mathrm{A}}\left|\exp \left(-T_{\mathrm{A}}\right) D_{\mathrm{A}} \exp \left(T_{\mathrm{A}}\right)\right| \mathrm{HF}_{\mathrm{A}}\right\rangle \\
= & \langle k|D| 0\rangle^{\mathrm{A}}
\end{aligned}
$$

and so the right transition moments are size-intensive. For the left transition moment in Eq. (20) we obtain

$$
\begin{aligned}
\langle 0|C| k\rangle= & \sum_{\mu_{\mathrm{A}}}\left\langle\Lambda\left|\left[C_{\mathrm{A}}+C_{\mathrm{B}}, \tau_{\mu_{\mathrm{A}}}\right]\right| \mathrm{CC}\right\rangle X_{\mu_{\mathrm{A}} k}^{R}+\sum_{\nu_{\mathrm{A}}} X_{v}^{C}\left(-\omega_{k}\right)\left\langle\Lambda\left|\left[\left[H_{\mathrm{A}}+H_{\mathrm{B}}, \tau_{\mu_{\mathrm{A}}}\right], \tau_{v}\right]\right| \mathrm{CC}\right\rangle X_{\mu_{\mathrm{A}} k}^{R} \\
= & \left(\left\langle\mathrm{HF} F_{\mathrm{A}}\left|+\sum_{v_{\mathrm{A}}} \zeta_{v_{\mathrm{A}}}\left\langle\nu_{\mathrm{A}}\right|\right) \exp \left(-T_{\mathrm{A}}\right)\left[C_{\mathrm{A}}, H_{\mathrm{A}}\right] \exp \left(T_{\mathrm{A}}\right) \mid \mathrm{HF}_{\mathrm{A}}\right\rangle\right. \\
& +\sum_{v_{\mathrm{A}} \mu_{\mathrm{A}}} X_{v_{\mathrm{A}}}^{C}\left(\omega_{k}\right)\left\langle\Lambda\left|\left[\left[H_{\mathrm{A}}, \tau_{\mu_{\mathrm{A}}}\right] \tau_{v_{\mathrm{A}}}\right]\right| \mathrm{CC}\right\rangle X_{\mu_{\mathrm{A}} k}^{R}=\langle 0|C| k\rangle^{\mathrm{A}}
\end{aligned}
$$

and therefore the left transition moment is also sizeintensive. Following an outline similar to the one for the transition moments, it is straightforward to show that CCLR second-order molecular properties are size extensive, i.e.,

$$
\langle\langle C ; D\rangle\rangle_{\omega}=\left\langle\left\langle C_{\mathrm{A}} ; D_{\mathrm{A}}\right\rangle\right\rangle_{\omega}+\left\langle\left\langle C_{\mathrm{B}} ; D_{\mathrm{B}}\right\rangle\right\rangle_{\omega}
$$

The nonseparability of the $\xi$ vector into components of the subsystems thus has no effect on the molecular property. This is so because the nonvanishing $\xi_{\mathrm{AB}}$ component always is multiplied by a zero component solution vector either by a right solution vector [Eq. (39)] or an eigenvector [Eq. (38)]\}.

\section{THE EQUATION OF MOTION COUPLED CLUSTER METHOD}

In a recent publication Stanton and Bartlett ${ }^{15}$ presented an equation of motion coupled cluster (EOMCC) method for the evaluation of excitation energies and transition moments. The excitation energies in the EOMCC and the CCLR approaches are identical, whereas the tran- 
sition moments differ. We describe these differences below and discuss some of the implications these differences may have for EOMCC transition moments. Initially, we write the EOMCC method using the notation of CCLR in Sec. II.
In EOMCC theory, the total energies of the excited states are determined by diagonalizing a Hamiltonian matrix in the biorthonormal basis defined by the left $\{\langle\mathrm{HF}| \exp (-T),\langle\mu| \exp (-T)\} \quad$ and right $\left\{\exp (T)|\mathrm{HF}\rangle, \tau_{\mu} \exp (T)|\mathrm{HF}\rangle\right\}$ basis vectors

$$
\begin{aligned}
\mathbf{H} & =\left(\begin{array}{cc}
\langle\mathrm{HF}|\exp (-T) H \exp (T)| \mathrm{HF}\rangle & \left\langle\mathrm{HF}\left|\exp (-T) H \exp (T) \tau_{v}\right| \mathrm{HF}\right\rangle \\
\langle\mu|\exp (-T) H \exp (T)| \mathrm{HF}\rangle & \left\langle\mu\left|\exp (-T) H \exp (T) \tau_{v}\right| \mathrm{HF}\right\rangle
\end{array}\right) \\
& =\left(\begin{array}{cc}
E_{\mathrm{CC}} & \left\langle\mathrm{HF}\left|\exp (-T) H \exp (T) \tau_{v}\right| \mathrm{HF}\right\rangle \\
0 & \left\langle\mu\left|\exp (-T) H \exp (T) \tau_{v}\right| \mathrm{HF}\right\rangle
\end{array}\right)=\left(\begin{array}{cc}
E_{\mathrm{CC}} & -\eta_{v} \\
0 & A_{\mu \nu}+\delta_{\mu v} E_{\mathrm{CC}}
\end{array}\right)
\end{aligned}
$$

where

$$
E_{\mathrm{CC}}=\langle\mathrm{HF}|\exp (-T) H \exp (T)| \mathrm{HF}\rangle=\langle\mathrm{HF}|H \exp (T)| \mathrm{HF}\rangle
$$

is the coupled cluster total energy, and where the zero elements occur because the cluster amplitudes satisfy the coupled cluster equation [Eq. (7)]. The vector components $\eta_{v}$ are defined in Eq. (12) and we have used

$$
\begin{aligned}
\left\langle\mu\left|\exp (-T) H \exp (T) \tau_{v}\right| \mathrm{HF}\right\rangle-\delta_{\mu v} E_{\mathrm{CC}} & =\left\langle\mu\left|\exp (-T) H \exp (T) \tau_{v}\right| \mathrm{HF}\right\rangle-\left\langle\mu\left|\tau_{v} H \exp (T)\right| \mathrm{HF}\right\rangle \\
& =\left\langle\mu\left|\exp (-T) H \exp (T) \tau_{v}\right| \mathrm{HF}\right\rangle-\left\langle\mu\left|\exp (-T) \tau_{v} H \exp (T)\right| \mathrm{HF}\right\rangle \\
& =\left\langle\mu\left|\exp (-T)\left[H, \tau_{v}\right] \exp (T)\right| \mathrm{HF}\right\rangle=A_{\mu \nu} .
\end{aligned}
$$

In order to insert $\exp (-T)$ in the second term after the second equality sign, we have used the $\mathrm{CC}$ amplitude equations [Eq. (7)]. Equation (47) has $E_{\mathrm{CC}}$ as an eigenvalue. Furthermore, the eigenvalues of the Jacobian $A_{\mu \nu}$ added to the total energy $E_{\mathrm{CC}}$ are eigenvalues of Eq. (47). These eigenvalues are the total energy $E_{k}$ of the excited states $|k\rangle$.

In EOMCC theory the transition moments are determined from the transition strength, which in a pure state picture is

$$
\langle 0|C| k\rangle\langle k|D| 0\rangle \text {. }
$$

The CC Hamiltonian matrix in Eq. (47) is nonsymmetric and the left and right eigenstates [the left and right eigenvectors of Eq. (47)] therefore differ. The state $|0\rangle$ representing the right eigenvector corresponding to the eigenvalue, $E_{\mathrm{CC}}$ becomes the coupled cluster state

$$
|0\rangle=|\mathrm{CC}\rangle=\exp (T)|\mathrm{HF}\rangle \text {. }
$$

The corresponding left eigenstate is identical to the state $\langle\Lambda|$ in Eq. (9):

$$
\langle 0|=\langle\Lambda|=\left(\langle\mathrm{HF}|+\sum_{v} \zeta_{v}\langle v|\right) \exp (-T),
$$

where we have used the normalization condition

$$
\langle\Lambda \mid \mathrm{CC}\rangle=1 \text {. }
$$

The eigenvectors of Eq. (47) are the same as the eigenvectors of the Jacobian [Eqs. (16) and (17)] except that a reference state component is used in the EOMCC method to identify the excited states $\langle k|$ and $|k\rangle$ :

$$
\begin{aligned}
|k\rangle & =\sum_{\mu}\left(\tau_{\mu} \exp (T)|\mathrm{HF}\rangle X_{\mu k}^{R}+\eta_{\mu} X_{\mu k}^{R} / \omega_{k}\right) \\
& =\sum_{\mu}\left(\tau_{\mu} \exp (T)|\mathrm{HF}\rangle X_{\mu k}^{R}-\zeta_{\mu} X_{\mu k}^{R}\right) \\
\langle k| & =\sum_{\mu} X_{\mu}^{L}\langle\mu| \exp (-T)
\end{aligned}
$$

where we have used Eq. (10). The excited states satisfy the normalization in Eq. (18). The transition moments in the EOMCC method therefore become

$$
\begin{aligned}
\langle k|D| 0\rangle= & \sum_{\mu} X_{\mu k}^{L}\langle\mu|\exp (-T) D| \mathrm{CC}\rangle, \\
\langle 0|C| k\rangle= & \sum_{\mu}\left(\left\langle\Lambda\left|C \tau_{\mu}\right| \mathrm{CC}\right\rangle X_{\mu k}^{R}\right. \\
& \left.-\zeta_{\mu} X_{\mu k}^{R}\langle\Lambda|C| \mathrm{CC}\rangle\right) .
\end{aligned}
$$

Comparison of the CCLR transition moments [Eqs. (19) and (20)] and the EOMCC transition moments shows that the right transition moment $\langle k|D| 0\rangle$ is identical in the two approaches. The left transition moment is different. In the limit where no truncation is carried out in the projection manifold (the FCI limit), the two approaches give identical transition moments. To see this, we write the CCLR left transition moment in Eq. (20) in the diagonal representation [see Eq. (102) of Ref. 12] 


$$
\begin{aligned}
\langle 0|C| k\rangle= & \sum_{\mu}\left\langle\Lambda\left|C \tau_{k}\right| \mathrm{CC}\right\rangle-\sum_{\mu}\left\langle\Lambda\left|\tau_{k} C\right| \mathrm{CC}\right\rangle \\
& -\sum_{n}\langle n|A| \mathrm{CC}\rangle\left(\omega_{n}+\omega_{k}\right)^{-1} \\
& \times\left\langle\Lambda\left|\left[\left[H, \tau_{k}\right], \tau_{n}\right]\right| \mathrm{CC}\right\rangle .
\end{aligned}
$$

In Eq. (58) we have used the notation

$$
\tau_{k}=\sum_{\mu} \tau_{\mu} X_{\mu k}^{R}
$$

The matrix element in the last term in Eq. (58) can be written as

$$
\begin{aligned}
\left\langle\Lambda\left|\left[\left[H, \tau_{k}\right], \tau_{n}\right]\right| \mathrm{CC}\right\rangle \\
=\left\langle\Lambda\left|H \tau_{n} \tau_{k}-\tau_{n} H \tau_{k}+\tau_{k} \tau_{n} H-\tau_{k} H \tau_{n}\right| \mathrm{CC}\right\rangle \\
=-\left(\omega_{n}+\omega_{k}\right)\left(\left\langle\Lambda\left|\tau_{k} \tau_{n}\right| \mathrm{CC}\right\rangle\right. \\
\left.\quad+\left(\sum_{\mu} \xi_{\mu} X_{\mu n}^{R}\right)\left(\sum_{\mu} \xi_{\mu} X_{\mu k}^{R}\right)\right),
\end{aligned}
$$

where we have used

$$
\begin{aligned}
& H|\mathrm{CC}\rangle=E_{\mathrm{CC}}|\mathrm{CC}\rangle, \\
& \langle\Lambda| H=E_{\mathrm{CC}}\langle\Lambda|,
\end{aligned}
$$

and

$$
\begin{aligned}
& H\left\{\tau_{k}|\mathrm{CC}\rangle+|\mathrm{CC}\rangle\left(\sum_{\mu} \xi_{\mu} X_{\mu k}\right)\right\} \\
& =E_{k}\left\{\tau_{k}|\mathrm{CC}\rangle+|\mathrm{CC}\rangle\left(\sum_{\mu} \zeta_{\mu} X_{\mu k}\right)\right\}
\end{aligned}
$$

which follows from the eigenvalue equation in Eq. (47). Inserting Eq. (60) into Eq. (58) and using the resolution of the identity

$$
\begin{gathered}
1=|\mathrm{CC}\rangle\left\langle\Lambda\left|+\sum_{n}\left\{\left(\sum_{\mu} \zeta_{\mu} X_{\mu n}^{R}\right)+\tau_{n}\right\}\right| \mathrm{CC}\right\rangle \\
\times\langle\mathrm{HF}| \tau_{n}^{+} \exp (-T)
\end{gathered}
$$

in the term $\left\langle\Lambda\left|\tau_{k} \tau_{n}\right| C C\right\rangle$ then straightforwardly gives that Eq. (58) can be written as Eq. (57). The left transition moment in EOMCC and CCLR theory therefore is identical in the FCI limit. For truncated manifolds the left transition moments in EOMCC and CCLR differ. The left transition moment in the EOMCC formalism can be written as

$$
\begin{aligned}
\langle 0|C| k\rangle= & \sum_{\mu}\left\langle\Lambda\left|\left[C, \tau_{\mu}\right]\right| C \mathrm{C}\right\rangle X_{\mu k}^{R} \\
& +\sum_{\mu}\left\langle\Lambda\left|\tau_{\mu} C\right| C \mathrm{C}\right\rangle X_{\mu k}^{R} \\
& -\left(\sum_{\mu} \zeta_{\mu} X_{\mu k}^{R}\right)\langle\Lambda|C| C \mathrm{C}\rangle,
\end{aligned}
$$

where the first term is identical to the first term in the CCLR expression [Eq. (20)]. The last two terms become

$$
\begin{aligned}
\sum_{\mu}\left\langle\Lambda\left|\tau_{\mu} C\right| C C\right\rangle X_{\mu k}^{R}-\left(\sum_{\mu} \zeta_{\mu} X_{\mu k}^{R}\right)\langle\Lambda|C| C C\rangle \\
=\sum_{\mu>v} \zeta_{\mu} X_{v k}^{R}\left\langle\mu\left|\tau_{\nu} \exp (-T) C\right| C C\right\rangle \\
\quad-\sum_{\mu}\left(\zeta_{\mu} X_{\mu k}^{R}\right) \sum_{v} \zeta_{\nu}\langle v|\exp (-T) C| C C\rangle
\end{aligned}
$$

The first term in Eq. (65) is size-intensive, whereas the correction [Eq. (66)] is not. The transition moments in EOMCC theory therefore are not size-intensive.

The identification of the transition moment in the EOMCC method relies on a configuration interaction interpretation of the states. In deriving the expressions for the transition matrix elements in Eqs. (56) and (57), it has been assumed that

$$
\begin{aligned}
& \langle k \mid 0\rangle=0, \\
& \langle k|H| 0\rangle=0,
\end{aligned}
$$

which is satisfied through the $\mathrm{CC}$ amplitude equations. Furthermore, it is assumed that

$$
\begin{aligned}
& \langle 0 \mid k\rangle=0, \\
& \langle 0|H| k\rangle=0,
\end{aligned}
$$

which is obtained by the diagonalization of Eq. (47). The right transition moment $\langle k|C| 0\rangle$ is the same in EOMCC

TABLE I. Dipole transition moments in EOMCC, CCLR, and RPA for the lowest totally symmetric excitation for $1,2, \ldots, N$ noninteracting LiH molecules. The internuclear distance of each LiH is 4 a.u.

\begin{tabular}{cccccccc}
\hline \hline \multirow{2}{*}{ Method } & $\begin{array}{c}\text { Number of } \\
\text { systems }\end{array}$ & $\langle 1|z| 0\rangle$ & $\langle 0|z| 1\rangle$ & $Y^{\mathrm{a}}$ & $X^{\mathrm{b}}$ & $U^{\mathfrak{c}}$ & $V^{\mathrm{d}}$ \\
\hline EOMCC & 1 & 2.436 & 1.888 & 2.3790 & -0.3898 & 0.2041 & 0.4949 \\
& 2 & 2.436 & 1.781 & 2.3790 & -0.3963 & 0.2041 & 0.9898 \\
& 3 & 2.436 & 1.673 & 2.3790 & -0.4028 & 0.2041 & 1.4846 \\
& 4 & 2.436 & 1.566 & 2.3790 & -0.4093 & 0.2041 & 1.9795 \\
CCLR & 5 & 2.436 & 1.458 & 2.3790 & -0.4158 & 0.2041 & 2.4744 \\
RPA & $N$ & 2.436 & 1.887 & & & & \\
\hline \hline
\end{tabular}

${ }^{\mathrm{a}} Y=\Sigma_{\mu}\left\langle\Lambda\left|\left[z, \tau_{\mu}\right]\right| \mathrm{CC}\right\rangle X_{\mu \mathrm{l}}^{R}$.

${ }^{\mathrm{b}} X=\Sigma_{\mu} \xi_{\mu} X_{\nu 1}^{R}\left\langle\mu\left|\tau_{v} \exp (-T) z\right| \mathrm{CC}\right\rangle$.

${ }^{\mathrm{c}} U=\Sigma_{\mu} \zeta_{\mu} X_{\mu 1}^{R}$.

${ }^{d} V=\Sigma \zeta_{v}\langle v|\exp (-T) z| C C)$. 


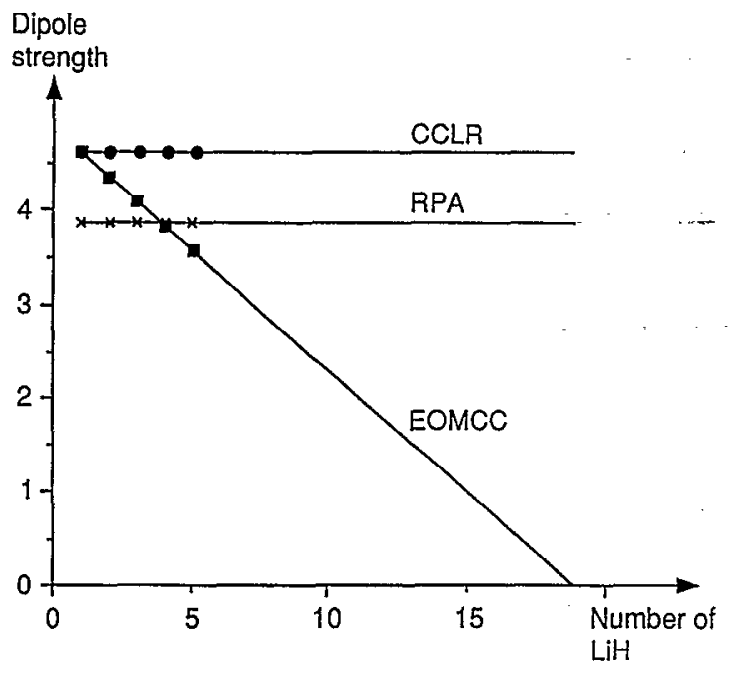

FIG. 1. The dipole strength as a function of the number of noninteracting $\mathrm{LiH}$ systems within the CCLR, RPA, and EOMCC approaches. Calculations have explicitly been carried out for up to five systems.

and CCLR as the EOM identification only relies on Eqs. (67) and (68). The differences in the left transition moment $\langle 0|D| k\rangle$ in EOMCC and CCLR is because Eqs. (69) and (70) are satisfied in EOMCC theory through diagonalizing Eq. (47). In CCLR, Eqs. (69) and (70) are a consequence of the fundamental assumption of linear response theory.

\section{RESULTS}

We describe CCLR and EOMCC singles and doubles calculations of excitation energies and dipole transition strengths for some of the lowest transitions from the ground states of $\mathrm{LiH}, \mathrm{CH}^{+}$, and $\mathrm{C}_{4}$. For $\mathrm{LiH}$ we consider calculations of the lowest totally symmetric transition for a sequence of noninteracting $\mathrm{LiH}$ systems to investigate the importance of using a size-intensive model. The $\mathrm{CH}^{+}$and $\mathrm{C}_{4}$ systems are considered because these molecules have previously been used in CCLR and EOMCC test calculations. For $\mathrm{CH}^{+}$full configuration interaction (FCI) results exist, ${ }^{18}$ and these have been used to judge the accuracy of the CCLR results.

The $\mathrm{LiH}$ calculations were carried out at an internuclear distance 4 a.u. using the double zeta basis of Dunning. ${ }^{19}$ The SCF and CCSD total energies were -7.94508 and -7.98747 a.u., respectively. For $\mathrm{CH}^{+}$we used the basis set and geometry of the FCI calculation. ${ }^{18}$ The $\mathrm{C}_{4}$ calculations were carried out at the rhombic geometry given in Ref. 20 and using the double zeta basis given in Ref. 20. The SCF and CCSD total energies were -151.16480 and -151.70878 a.u., respectively.

In Table I we give the dipole transition moments for the lowest totally symmetric dipole allowed transition in CCLR and EOMCC singles and doubles calculations for a sequence of noninteracting $\mathrm{LiH}$ systems. For comparison we also give the transition moments in the random phase
TABLE II. CCLR, EOMCC, and FCI excitation energies and dipole strengths for the lowest transitions in $\mathrm{CH}^{+}$.

\begin{tabular}{|c|c|c|c|c|c|c|}
\hline \multicolumn{3}{|c|}{ Excitation energy } & \multicolumn{4}{|c|}{ Dipole strength } \\
\hline $\begin{array}{l}\text { Final } \\
\text { state }\end{array}$ & CCLR & FCI & CCLR & EOMCC & FCI & $\% t_{1}$ \\
\hline${ }^{1} \Sigma^{+}$ & 9.1089 & 8.5492 & 0.0247 & 0.025 & 0.025 & 0.4 \\
\hline${ }^{1} \Sigma^{+}$ & 13.5805 & 13.5246 & 1.0752 & 1.073 & 1.080 & 92.9 \\
\hline${ }^{1} \Sigma^{+}$ & 17.3157 & 17.217 & 0.7194 & 0.720 & 0.684 & 86.1 \\
\hline${ }^{1} \Pi$ & 3.2607 & 3.2296 & 0.0943 & 0.095 & 0.090 & 97.0 \\
\hline${ }^{1} \Pi$ & 14.4544 & 14.1271 & 0.6867 & 0.692 & 0.588 & 77.4 \\
\hline
\end{tabular}

approximation (RPA). The excitation energies are sizeintensive in the CCLR, EOMCC, and RPA model. The RPA excitation energy is $5.072 \mathrm{eV}$ and for CCLR the excitation energy is $4.355 \mathrm{eV}$ which is identical to the EOMCC excitation energy. CCLR and RPA give sizeintensive transition moments; the transition moments are therefore only given for $N$ noninteracting $\mathrm{LiH}$ systems for these two approaches. RPA is a variational approach and the left $(\langle 0|z| 1\rangle)$ and right $(\langle 1|z| 0\rangle)$ transition moments are therefore identical. The CCLR and EOMCC approaches are nonvariational and the left and right transition moments differ. The right transition moment is the same in CCLR and EOMCC; only the left transition moment differs. The left transition moment is not sizeintensive in the EOMCC approach and is given in Table I for one to five noninteracting $\mathrm{LiH}$ systems. The EOMCC left transition moment is evaluated as described in Eq. (65) where the first term ( $X$ in Table $I$ ) is size-intensive, the second term [ $Y$ in Table $I$ and the first term in Eq. (66)] contains terms that are not size-intensive, and the last term in Eq. (66) consists of a product of two terms $(-U V)$ where $U$ is size-intensive and $V$ is size-extensive. For a single LiH system EOMCC and CCLR give very much the same left transition moment. When the number of noninteracting LiH systems increases, the EOMCC left transition moment decreases, while the CCLR left transition moment is unchanged. The major reason for the decrease of the left transition moment is the term $(-U V)$. The factor $U$ is size-intensive while $V$ is size-extensive. For example, for five systems $V$ is exactly five times $V$ of a single system. As a result the EOMCC left transition moment decreases rapidly towards zero with increasing number of noninteracting systems. Term $X$ contains both sizeextensive and size-intensive terms. The size-extensive terms substantiate further the decrease in the left transition moment as the number of LiH systems increases. An extrapolation of the results in Table I shows that for 19 or more noninteracting $\mathrm{LiH}$ systems the left transition moment becomes negative. This leads to the absurd result of a negative transition strength. In Fig. 1 we have plotted the dipole transition strengths in the CCLR, EOMCC, and RPA approaches for an increasing number of noninteracting LiH molecules to emphasize which approaches give sizeintensive transition moments and which do not.

In Table II we give the excitation energies and dipole transition strengths for some of the lowest dipole allowed 
TABLE III. Comparison of CCLR, EOMCC, and MRDCI excitation energies and oscillator strength for rhombic $\mathrm{C}_{4}$ for the lowest dipole allowed transitions. The molecule is oriented in the $x y$ plane with the longer $\mathrm{CC}$ distance coincident with the $x$ axis.

\begin{tabular}{|c|c|c|c|c|c|c|c|}
\hline & \multicolumn{3}{|c|}{ Excitation energies $(\mathrm{eV})$} & \multicolumn{4}{|c|}{ Oscillator strength } \\
\hline & CCLR & EOMCC & MRDCI & CCLR & EOMCC & MRDCI & $\% t_{1}$ \\
\hline${ }^{1} B_{1 u}$ & 2.450 & 2.46 & 2.39 & 0.015 & 0.016 & 0.025 & 90.1 \\
\hline${ }^{1} B_{2 u}$ & 6.939 & 6.94 & 7.03 & 0.110 & 0.114 & 0.836 & 89.8 \\
\hline${ }^{1} B_{3 u}$ & 6.889 & 6.90 & 6.88 & 0.089 & 0.094 & 0.183 & 89.4 \\
\hline
\end{tabular}

transitions in $\mathrm{CH}^{+}$. We also report the $\% t_{1}$ amplitudes in the right eigenvectors. The EOMCC transition strengths have previously been reported by Stanton and Bartlett. ${ }^{15}$ The CCLR and EOMCC transition strengths are very similar to the CCLR results, being slightly closer to the FCI results. For excitations dominated by $t_{1}$ amplitudes the CCLR results are in good agreement with FCI results. This is so because the excited states which have dominating single excitation components (small $\% t_{2}$ ) can be correlated reasonably well by the double excitation.

In Table III we report CCLR, EOMCC, and multireference configuration interaction (MRDCI) ${ }^{20}$ excitation energies and oscillator strengths for the three lowest dipole allowed transitions. The EOMCC results have also previously been reported by Stanton and Bartlett ${ }^{15}$ and MRDCI results by Pachioni and Koutecky. ${ }^{20}$ We assign the small difference in the CCLR and EOMCC excitation energies to small differences in the used geometries in the two calculations. The CCLR and MRDCI excitation energies are very similar. The CCLR and EOMCC oscillator strengths are similar but differ substantially from the MRDCI oscillator strength. Since the $\% t_{1}$ amplitudes are large for all the transitions, the CCLR results are expected to be rather accurate.

\section{DISCUSSION}

Coupled cluster singles and doubles linear response (CCLR) calculations have been presented for excitation energies and transition strengths for the lowest dipole allowed transition for $\mathrm{LiH}, \mathrm{CH}^{+}$, and $\mathrm{C}_{4}$, and the results have been compared with corresponding equation of motion coupled cluster (EOMCC) results. The excitation energies in the CCLR and EOMCC approaches are identical and size-intensive. The transition strength matrix elements differ in the two approaches. We show CCLR gives sizeintensive transition moments, whereas the EOMCC approach does not. To illustrate the consequences this can have on the calculated transition strengths, we have carried out $\mathrm{LiH}$ calculations of the dipole transition strength for the lowest totally symmetric excitation for a sequence of noninteracting $\mathrm{LiH}$ systems. For a single $\mathrm{LiH}$ system, the CCLR and EOMCC give similar transition strengths. However, when the number of $\mathrm{LiH}$ systems was increased the transition strengths decreased in the EOMCC approach, while as expected it was unchanged in the CCLR approach. We found that for 19 or more noninteracting LiH systems the transition strength becomes negative. This is, of course, an absurd result that makes it questionable to use the EOMCC approach for larger systems, in spite of the fact that the test calculations on single smaller molecules $\left(\mathrm{LiH}, \mathrm{CH}^{+}\right.$, and $\mathrm{C}_{4}$ ) have all given close agreement between CCLR and EOMCC dipole transition strengths.

Frequency independent polarizabilities can for an exact state be written in terms of a sum over state expression. If the CCLR excitation energies and transition moments are used in this sum over state expression we obtain the polarizability of a finite field energy calculation with nonrelaxed orbitals. This formal consistency together with the fact that the CCLR model gives size-intensive transition strengths makes the CCLR approach a very attractive "black box" approach for calculations of accurate excitation energies and transition moments.

\section{ACKNOWLEDGMENTS}

We would like to thank Professor Jan Linderberg for helpful discussions. This work has been supported by the Danish Natural Science Research Council (Grant No. 119004).

${ }^{1} \mathrm{~J}$. Linderberg and Y. Öhrn, Propagators in Quantum Chemistry (Academic, New York, 1973).

${ }^{2} \mathrm{P}$. J $\phi$ rgensen and J. Simons, Second Quantization Based Methods in Quantum Chemistry (Academic, New York, 1981).

${ }^{3}$ A. D. McLachlan and A. Ball, Rev. Mod. Phys. 36, 844 (1964).

${ }^{4}$ D. L. Yeager and P. Jørgensen, Chem. Phys. Lett. 65, 77 (1979).

${ }^{5}$ H. J. Monkhorst, Int. J. Quantum Chem. Symp. 11, 421 (I977).

${ }^{6}$ E. Dalgaard and H. J. Monkhorst, Phys. Rev. A 28, 1217 (1983).

${ }^{7}$ E. S. Nielsen, P. J $\phi$ rgensen, and J. Oddershede, J. Chem. Phys. 73, 6238 (1980).

${ }^{8}$ P. E. S. Wormer and W. Rijks, Phys. Rev. A 33, 2928 (1986).

${ }^{9}$ J. E. Rice and N. C. Handy, J. Chem. Phys. 94, 4959 (1991).

${ }^{10}$ See, for example, R. J. Bartlett, J. Phys. Chem. 93, 169 (1989).

${ }^{11}$ H. Sekino and R. J. Bartlett, J. Chem. Phys. 98, 3022 (1993).

${ }^{12} \mathrm{H}$. Koch and P. Jørgensen, J. Chem. Phys. 93, 3333 (1990).

${ }^{13} \mathrm{H}$. Koch, H. J. Aa. Jensen, P. Jørgensen, and T. Helgaker, J. Chem. Phys. 92, 4924 (1990).

${ }^{14}$ See, for example, P. W. Atkins, Physical Chemistry, 2nd ed. (Oxford University, Oxford, 1982), p. 76.

${ }^{15}$ J. F. Stanton and R. J. Bartlett, J. Chem. Phys. 98, 7029 (1993).

${ }^{16}$ R. Kobayashi, H. Koch, and P. Jørgensen, Chem. Phys. Lett. (submitted).

${ }^{17}$ H. Koch, H. J. Aa Jensen, P. Jørgensen, T. Helgaker, G. E. Scuseria, and H. F. Schaefer, J. Chem. Phys. 92, 4924 (1990).

${ }^{18}$ J. Olsen, A. M. Sanchez de Merás, H. J. Aa. Jensen, and P. Jørgensen, Chem. Phys. Lett. 154, 380 (1989).

${ }^{19}$ T. H. Dunning, J. Chem. Phys. 55, 716 (1971).

${ }^{20}$ G. Pacchioni and J. Koutecky, J. Chem. Phys. 88, 1066 (1988). 